\title{
Evidence that Osteocytes in Autogenous Bone Fragments can Repair Disrupted Canalicular Networks and Connect with Osteocytes in de novo Formed Bone on the Fragment Surface
}

\author{
Furqan A. Shah ${ }^{1,2} \cdot$ Anders Palmquist ${ }^{1,2}$
}

Received: 6 March 2017/ Accepted: 20 April 2017/Published online: 10 May 2017

(C) The Author(s) 2017. This article is an open access publication

\begin{abstract}
Autogenous bone fragments generated during surgery (e.g. implant site preparation) accelerate bone formation by the release of a large variety of growth factors from the extracellular matrix and the cells contained within. Osteocytes, whether viable or apoptotic, within such fragments are able to recruit osteoclasts to a site of bone remodelling. Here, using correlative scanning electron microscopy, we provide compelling evidence that at one week healing in the Sprague Dawley rat tibia, following surgery (and/or the placement of a bone-anchored implant), autogenous bone fragments support bone formation on their surface. Furthermore, osteocytes within the autogenous fragments are frequently able to repair the disrupted canalicular networks and appear to connect with osteocytes (or osteoblastic-osteocytes) in the de novo formed bone on the surface of the fragment.
\end{abstract}

Keywords Autogenous bone $\cdot$ Early implant healing · Osteocyte $\cdot$ Scanning electron microscopy

\section{Introduction}

Autogenous bone, still considered the gold standard for most bone graft applications, osseointegrates with the surrounding bone and undergoes vascularisation [1],

Furqan A. Shah

furqan.ali.shah@biomaterials.gu.se

1 Department of Biomaterials, Institute of Clinical Sciences, Sahlgrenska Academy at University of Gothenburg, Göteborg, Sweden

2 BIOMATCELL VINN Excellence Center of Biomaterials and Cell Therapy, Göteborg, Sweden and is believed to provide optimal osteoconductive, osteoinductive, and osteogenic properties [2]. A number of growth factors affecting bone formation and resorption may be released from the extracellular matrix and cells (e.g. osteoblasts) in autogenous bone grafts including transforming growth factor beta (TGF- $\beta$ ) and bone morphogenetic protein (BMP), fibroblast growth factor (FGF), insulin-like growth factor (IGF), plateletderived growth factor (PDGF) [3], vascular endothelial growth factor (VEGF), receptor activator of nuclear factor kappa- $\beta$ ligand (RANKL), and osteoprotegerin (OPG) [4]. Osteoclasts on the surface of autogenous fresh rib grafts are observed in close contact with the cytoplasmic processes of apparently viable osteocytes within the graft [5]. Viable osteocytes have also been reported within a microvascular fibula flap used in combination with an additively manufactured osteosynthesis plate after 33 months in the human mandible [6]. Both viable and apoptotic osteocytes can recruit osteoclasts to sites of bone remodelling [7]. In the context of peri-implant healing, it is claimed that autogenous bone fragments accelerate bone formation and offer the possibility of earlier implant loading [8]; however, bone fragments generated during implant site preparation are believed to be devoid of osteocytes $[9,10]$. While it may be true that the destructive nature of the drilling process could render osteocytes non-viable, we report compelling evidence that such an assumption may not hold true in all cases. Here, we demonstrate that osteocytes in autogenous bone fragments can potentially restore disrupted canalicular networks and connect with osteocytes in the bone formed on the surface of such fragments. 


\section{Materials and Methods}

Bone-implant blocks embedded in LR White resin (London Resin Company, UK) were obtained from in vivo animal experiments conducted previously in our group. All specimens represented an early healing stage, i.e. 6-7 days, following the placement of commercially pure (Grade 4) titanium (cp-Ti) implants with and without different commonly applied surface modifications in Sprague Dawley rat tibia. Resin embedded bone-implant blocks were polished
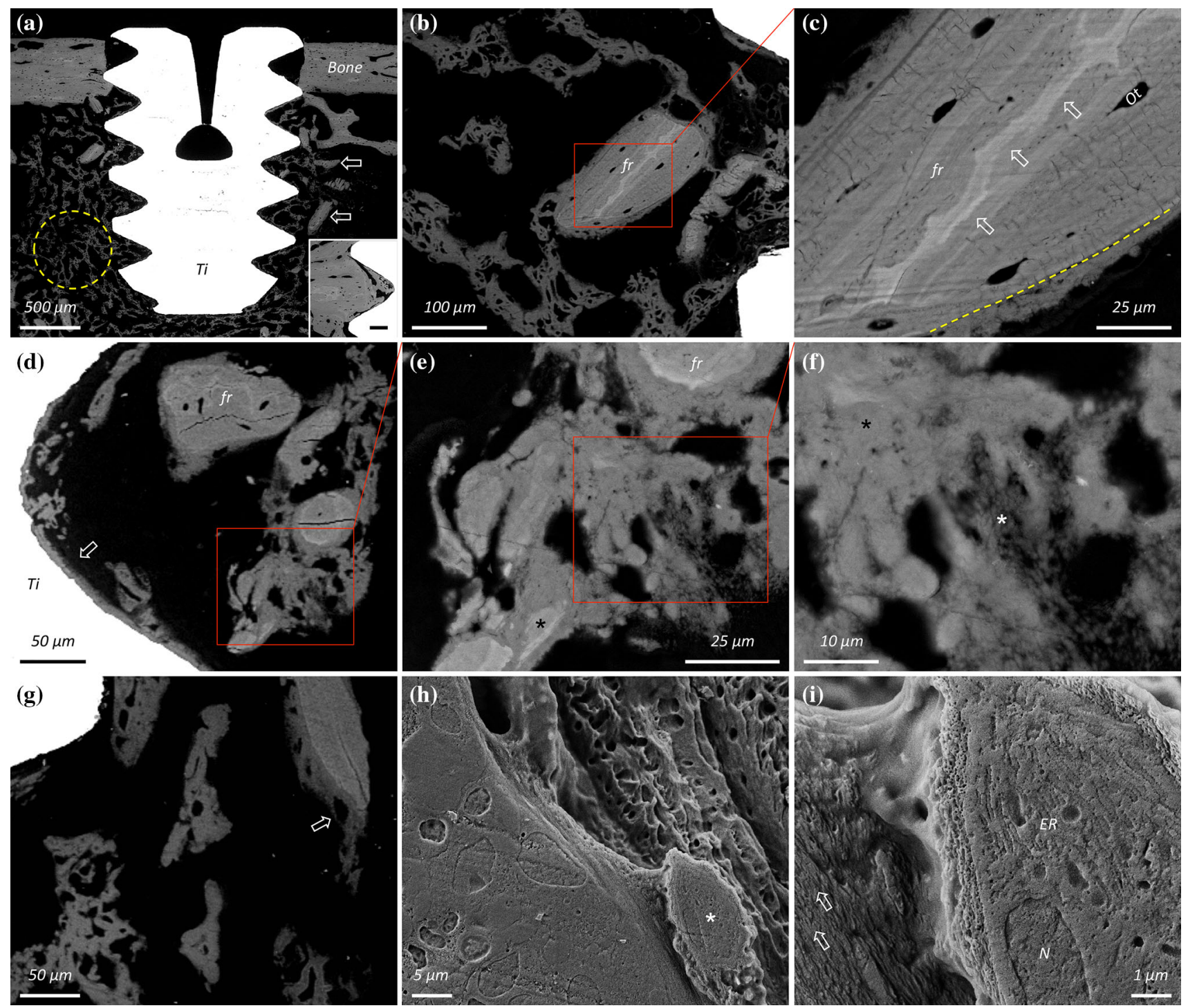

Fig. 1 a A screw-shaped, machined, cp-Ti implant (Ti) in Sprague Dawley rat tibia. At one week of healing, large amounts of rapidly formed woven bone (yellow ring) and autogenous bone fragments of varying dimensions (arrows) are observed within and around the implant threads. Inset: A similarly prepared implant surface at 28 days of healing (Scale bar $100 \mu \mathrm{m}$ ). b, c Fragments (fr) of the original cortical bone, identified in the rat by the presence of islands of unremodelled hypermineralised cartilage (arrows), behave as osteoconductive surfaces that support new bone formation. The interface between the autogenous bone fragment and the newly formed bone on the surface is clearly demarcated (yellow broken line) by the striking difference in the BSE Z-contrast. $\mathbf{d}$ In addition to bone apposition directly on the implant surface (arrow), autogenous bone fragments and woven bone are seen within the implant thread. e Woven bone lacks a well-ordered structure. However, tiny fragments of the original organised, lamellar bone (black asterisk) become embedded and incorporated within the new forming tissue. f Extracellular matrix at different stages of mineralisation can be distinguished by a variation in the BSE Z-contrast, where older and relatively more mature tissue (black asterisk) appears brighter than younger tissue (white asterisk). $\mathbf{g}$ New bone formation on a large fragment. h, i Following resin cast etching, the extracellular matrix deposited next to an osteoblastic-osteocyte (asterisk) on the autogenous bone fragment exhibits a well-aligned and ordered arrangement that is maintained up to 'cellular' length scales (arrows). The endoplasmic reticulum (ER) and nucleus (N) can be identified inside the osteoblastic-osteocyte 
using 400-4000 grit SiC paper and examined in a Quanta 200 environmental SEM (FEI Company, The Netherlands) operated in the backscattered electron (BSE) mode at $20 \mathrm{kV}$ accelerating voltage, 0.5 Torr water vapour pressure. Elemental analysis was performed using energy dispersive X-ray spectroscopy (INCA EDX system, Oxford Instruments $\mathrm{GmbH}$, Wiesbaden, Germany) performed at $15-20 \mathrm{kV}$ accelerating voltage, $10 \mathrm{~mm}$ working distance, $40 \mu \mathrm{m}$ aperture size, and $0-10 \mathrm{keV}$ spectral energy range. Elemental maps for the $\mathrm{K} \alpha \mathrm{X}$-ray emission lines for calcium $(\sim 3.691 \mathrm{keV})$, phosphorus $(\sim 2.013 \mathrm{keV})$, and carbon $(\sim 0.277 \mathrm{keV})$ are shown. The osteocyte lacunocanalicular network was exposed by resin cast etching for direct visualisation [11]. Briefly, the polished resin embedded bone-implant blocks were sequentially immersed in 9\% ortho-phosphoric acid and 5\% sodium hypochlorite. After overnight drying, the samples were Au sputter-coated $(10 \mathrm{~nm}$ ), and examined in an Ultra 55 FEG SEM (Leo Electron Microscopy Ltd, UK) operated in the secondary electron (SE) mode at $5 \mathrm{kV}$ accelerating voltage and $5 \mathrm{~mm}$ working distance.

\section{Results}

The early healing stage of bone around implanted titanium materials is characterised by the appearance of rapidly formed woven bone (Fig. 1a). Autogenous bone fragments, varying greatly in size and quantity, are frequently observed around the implant and also within the implant threads (Fig. 1b). In the laboratory rat, e.g. Sprague Dawley, fragments of the original cortical bone can be identified by the inclusion of islands of unremodelled hypermineralised cartilage [12]. Such fragments behave as osteoconductive surfaces that support and guide new bone formation (Fig. 1c). Autogenous fragments are also detected in close spatial association with rapidly forming woven bone (Fig. 1d). The extracellular matrix at different stages of mineralisation can be distinguished by a variation in the BSE Z- (atomic number) contrast, where younger (or less mature) tissue exhibits a lower $Z$-contrast and appears relatively darker due to lower local calcium content (Fig. 1e, f). In contrast to woven bone, partially embedded osteocytes in the new forming bone, also referred to as
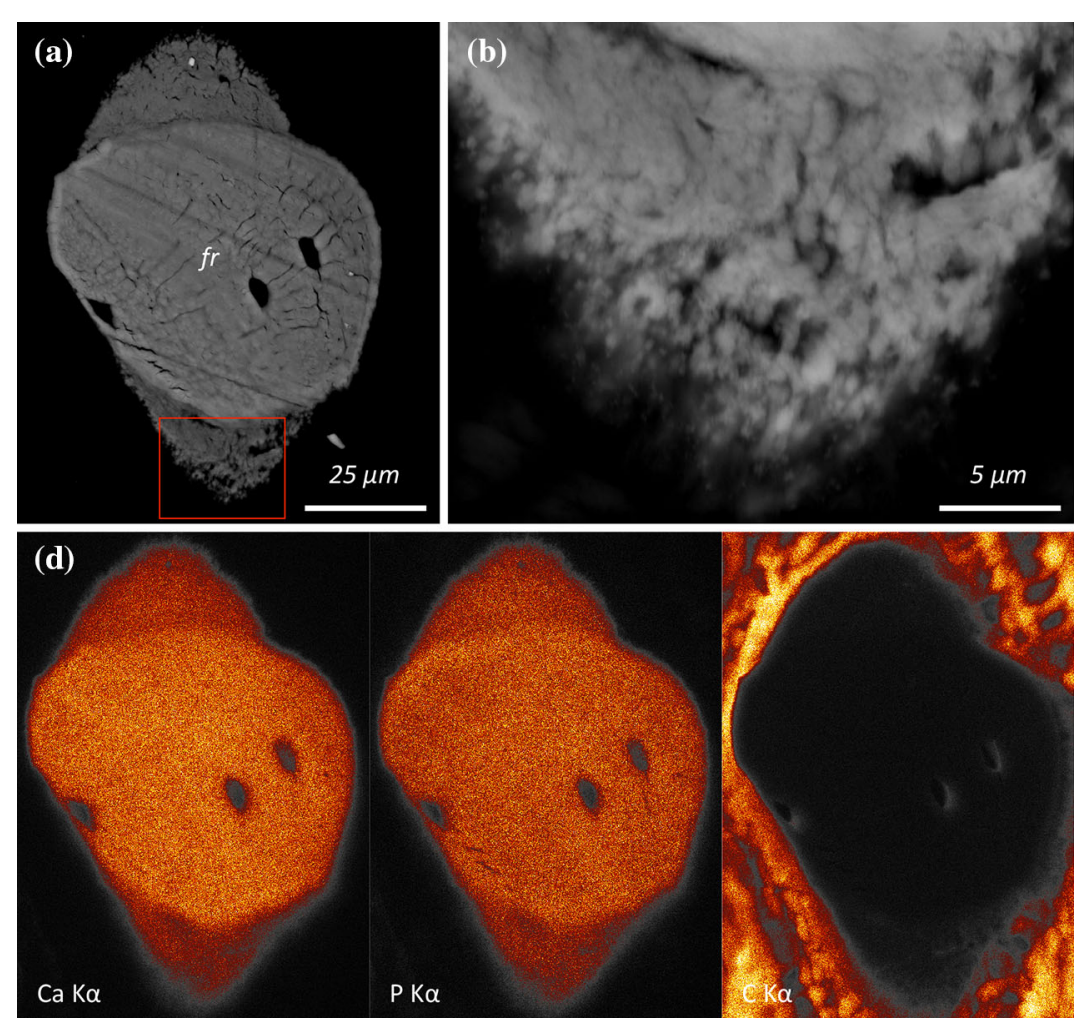

Fig. 2 a, b The mineralisation front appears granular. c The newly formed bone bridges the gap between two fragments. Inset: Detail of the granular mineralisation front (Scale bar $10 \mu \mathrm{m}$ ). d, e Elemental maps representing the $\mathrm{K} \alpha \mathrm{X}$-ray emission lines for calcium $(\mathrm{Ca} \mathrm{K} \alpha)$,

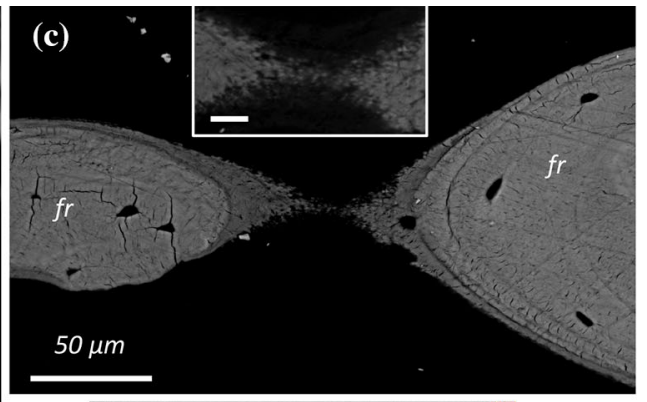

(e)

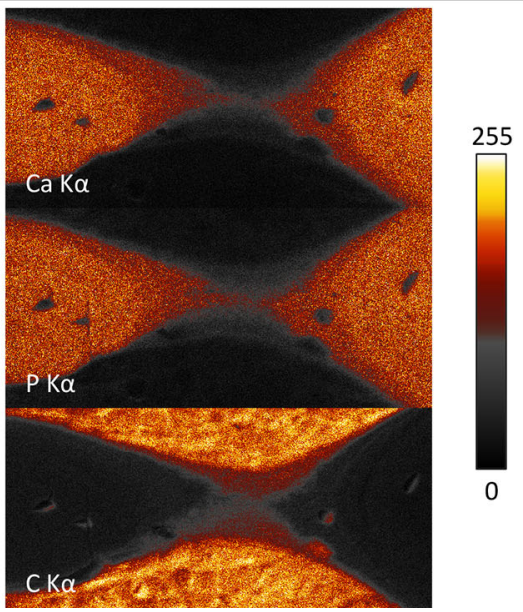

phosphorus (P K $\alpha$ ), and carbon $(\mathrm{C} \mathrm{K} \alpha)$ corresponding to (a) and (c), respectively, reveal lower $\mathrm{Ca}$ and $\mathrm{P}$ content of the newly formed bone compared to the old bone of the fragment 

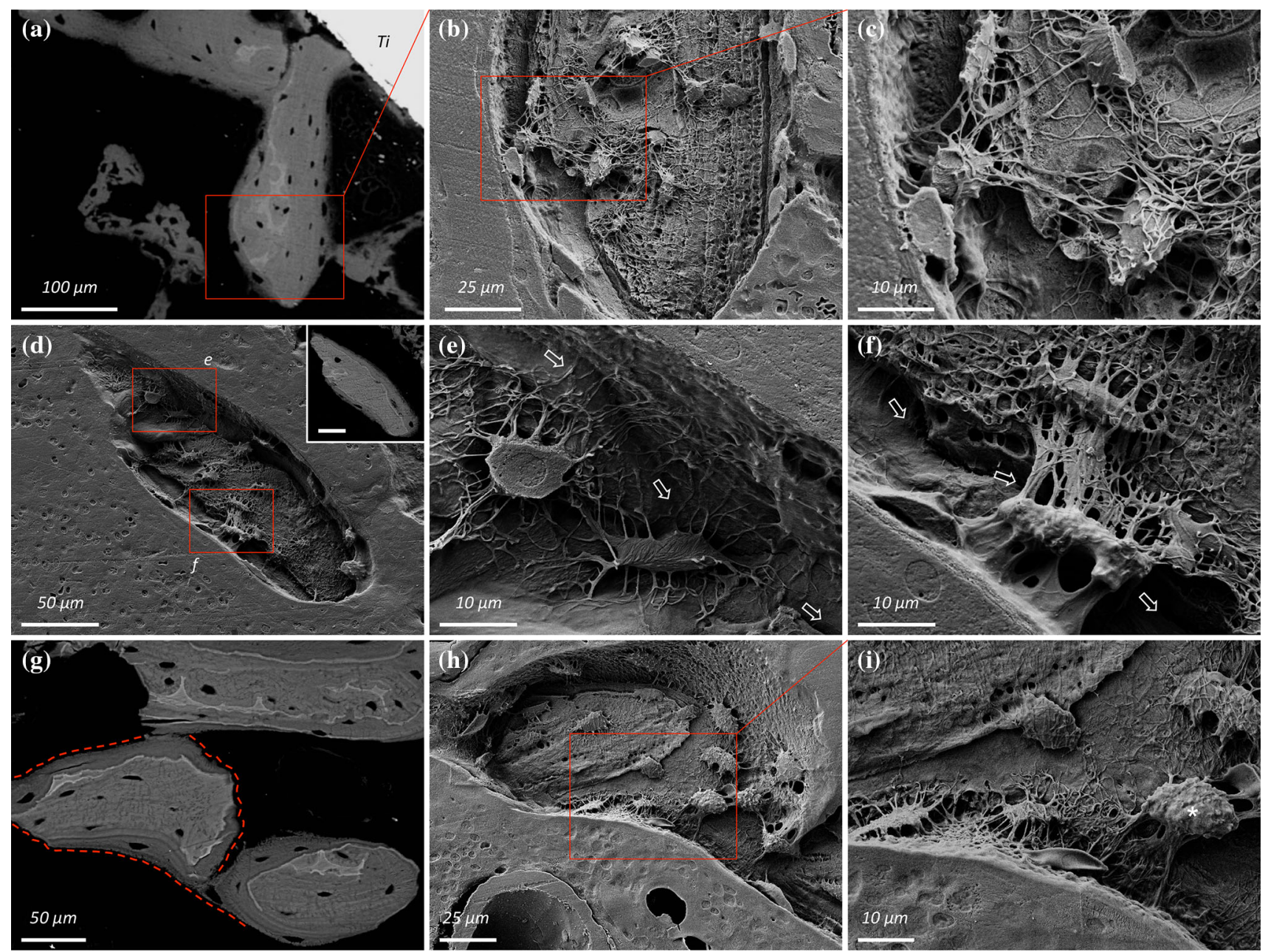

Fig. 3 a Example \#1: A large autogenous bone fragment within an implant thread (Ti) with newly formed bone on the surface. b, c Following resin cast etching, a high degree of interconnectivity is observed between osteocytes in the newly formed bone and those in the underlying fragment. d Example \#2: A large autogenous bone fragment outside the implant thread with newly formed bone on the surface. Inset: BSE-SEM image of the fragment (Scale bar $50 \mu \mathrm{m})$. e, f At regions indicated in (d), networks of interconnected resin-filled canaliculi cross the interface (arrows) between the old bone of the fragment and the newly formed bone on the surface, and extend up to the marrow space. g Example \#3: Several autogenous bone fragments in close proximity to each other. h, i Following resin cast etching, canaliculi from an osteocyte (asterisk) in one of the fragments extend towards the marrow space osteoblastic-osteocytes [13], are aligned parallel to the surface of the underlying fragment, and subsequently the extracellular matrix deposited on the fragment surface appears well aligned and ordered (Fig. 1g-i). The mineralisation front exhibits a granular appearance due to the presence of sub-micron sized foci (Fig. 2a, b), consistent with the description of mineralised spheres associated with the cellular processes of primary osteoblasts and of MLOA5 (postosteoblast/preosteocyte-like) cells [14]. When several fragments are located close together, the newly formed bone on their surface may bridge the intervening space (Fig. 2c). Elemental analysis, by EDX, confirms lower calcium and phosphorus content of the newly formed tissue on the fragment surface than the old bone of the fragments (Fig. 2d, e).
As bone formation proceeds, osteocytes within the autogenous bone fragments appear to interact physically with osteocytes in the de novo formed bone on the fragment surface. Canaliculi from the osteocytes residing within the old bone of the fragments extend beyond the original periphery and into the new forming tissue where they make numerous connections with newer osteocytes (Fig. 3).

Attributable to the inverse relationship between particle size and surface-to-volume ratio, small autogenous bone fragments present a large surface area for new bone formation (Fig. 4a, b). It is not infrequent to observe high coverage of the fragment's surface with newly formed bone, and even at this early healing stage, the newly formed bone occupies a large surface area. Moreover, by 

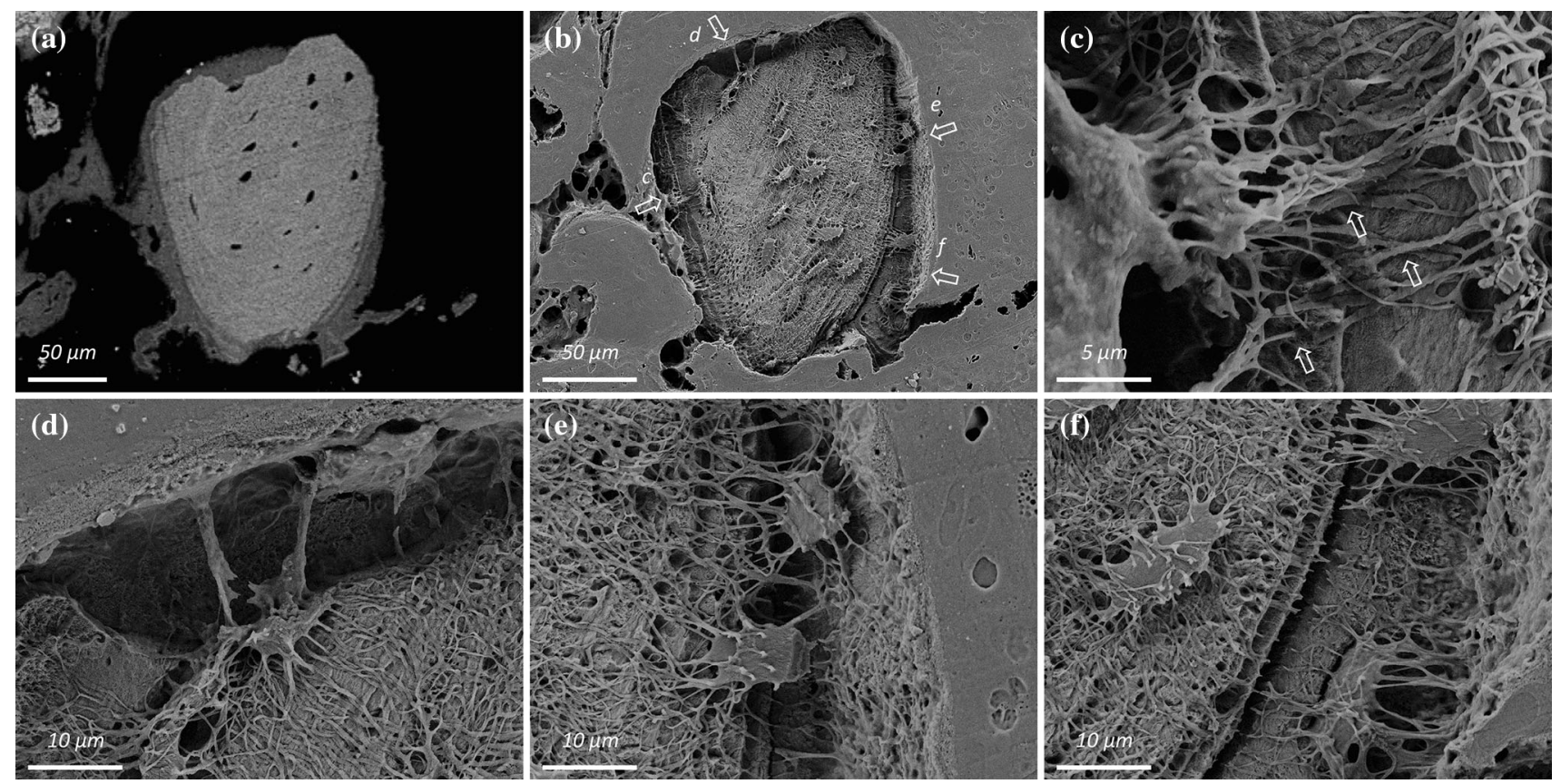

Fig. 4 a An autogenous bone fragment outside the implant thread that presents a large surface area for new bone formation. The newly formed bone covers approximately $75 \%$ of the surface, and occupies an area nearly $38 \%$ of the area of the underlying fragment. b-

virtue of a physical interaction between osteocytes within the old bone of the fragments and those in the newly formed bone, osteocytes residing deep within the fragments maintain, or alternatively, regain connectivity with the surrounding marrow space (Fig. 4c-f).

\section{Discussion and Conclusions}

Implant surfaces considered to exhibit a higher osteogenic potential display a higher degree of bone-implant contact or direct bone apposition, often referred to as contact osteogenesis. As a consequence of such an osteogenic potential, precursor cells, i.e. mesenchymal stem cells (MSCs) are recruited to an implant (or a surgical defect) site, resulting in high amounts of rapidly formed woven bone. This early-formed tissue, however, lacks a wellordered structure [15]. Gradually, this tissue is removed by osteoclastic activity and is replaced by organised, lamellar bone-a process that progresses at a considerably slower pace in comparison to the formation of woven bone.

Particulate, decellularised bone has been used as an effective scaffold for bone repair [16]. Small bone fragments in healing bone sites, generated during surgery, lend themselves to a unique scenario where they serve as osteoinductive surfaces and provide attachment sites for bone forming cells, i.e. osteoblasts. It is assumed that f Following resin cast etching, at regions indicated in (b), osteocytes in the autogenous bone fragment connect with those in the newly formed bone and thereby afford connectivity with the surrounding marrow space

canalicular networks associated with the osteocytes nearest to the fragment surface are disrupted and/or destroyed during the surgical procedure. Incorporation of autologous bone grafts occurs through a process termed creeping substitution (a slow, near-complete resorption of the graft with simultaneous deposition of new, viable bone) [17]. Contrary to the fate of cortical autografts, where mature osteocytes degenerate in the early stages following transplantation [18], when autogenous bone fragments are generated in the defect during the drilling process without exposure to an ex vivo environment, the osteocytes possibly remain viable and functional.

Here we demonstrate, using correlative scanning electron microscopy (SEM) techniques, that $(i)$ autogenous bone fragments contribute towards osteogenesis within healing surgical defects, e.g. in the vicinity of bone-anchored implants, and (ii) osteocytes within autogenous bone fragments are frequently observed to restore a close physical proximity with osteocytes (osteoblastic-osteocytes) in new bone formed on the surface of these fragments, through interconnecting canaliculi that contain cytoplasmic extensions of osteocytes. However, it is not known whether the restored interconnectivity between osteocyte canaliculi in old and new bone plays any role in transmitting biochemical signals or transporting biomolecules involved in osteocyte function. Although certain implant surfaces are believed to exhibit an enhanced 
osteogenic potential and stronger mechanical interlocking with the surrounding bone tissue $[19,20]$, it is assumed that the presence of autogenous bone fragments within a healing surgical defect, and the prevalence thereof, is a function of the surgical technique. Therefore, the physico-chemical properties of the implant surface have no direct bearing on the osteopromotive potential of such bone fragments. Nevertheless, bone drilling may induce osteocyte death, particularly as a function of time [21], and temperature [22].

Further experiments are required to ascertain the viability and the eventual fate of the osteocytes in autogenous bone fragments. Such information may be of benefit in optimising surgical and drilling techniques in order to minimise their destructive effects on the implantation site. The presence of bone fragments at experimental implantation sites, particularly within implant threads, and their osteogenic potential have wide-ranging implications on peri-implant healing. It may be appreciated that, at least during early healing, such autogenous bone fragments (and the associated de novo formed bone) contribute to the overall amount of mineralised tissue found within the healing defect in addition to bone apposition directly on the implant surface.

Acknowledgements This study was supported by the Swedish Research Council (Grant K2015-52X-09495-28-4), the BIOMATCELL VINN Excellence Center of Biomaterials and Cell Therapy, the Region Västra Götaland, the ALF/LUA Research Grant "Optimization of osseointegration for treatment of transfemoral amputees" (ALFGBG-448851), the IngaBritt and Arne Lundberg Foundation, the Dr. Felix Neubergh Foundation, Promobilia, the Hjalmar Svensson Foundation, the Wilhelm and Martina Lundgren Vetenskapsfond, and the Materials Science Area of Advance at Chalmers and the Department of Biomaterials, University of Gothenburg. The authors wish to thank Prof. Peter Thomsen for many helpful discussions and Dr. Sarunas Petronis for kindly providing the specimens used in this work.

\section{Compliance with Ethical Standards}

Conflict of interest Furqan A. Shah and Anders Palmquist declare that they have no conflict of interest.

Ethical Approval All applicable international, national, and/or institutional guidelines for the care and use of animals were followed.

Human and Animal Rights and Informed Consent All procedures performed in studies involving animals were in accordance with the ethical standards of the institution or practice at which the studies were conducted. This article does not contain any studies with human participants performed by any of the authors.

Open Access This article is distributed under the terms of the Creative Commons Attribution 4.0 International License (http://creative commons.org/licenses/by/4.0/), which permits unrestricted use, distribution, and reproduction in any medium, provided you give appropriate credit to the original author(s) and the source, provide a link to the Creative Commons license, and indicate if changes were made.

\section{References}

1. Rogers GF, Greene AK (2012) Autogenous bone graft: basic science and clinical implications. J Craniofacial Surg 23:323-327

2. Giannoudis PV, Dinopoulos H, Tsiridis E (2005) Bone substitutes: an update. Injury 36:S20-S27

3. Lieberman JR, Daluiski A, Einhorn TA (2002) The role of growth factors in the repair of bone. Biology and clinical applications. J Bone Joint Surg Am 84:1032-1044

4. Miron RJ, Gruber R, Hedbom E, Saulacic N, Zhang Y, Sculean A, Bosshardt DD, Buser D (2013) Impact of bone harvesting techniques on cell viability and the release of growth factors of autografts. Clin Implant Dent Relat Res 15:481-489

5. Kamijou T, Nakajima T, Ozawa H (1994) Effects of osteocytes on osteoinduction in the autogenous rib graft in the rat mandible. Bone 15:629-637

6. Thor A, Palmquist A, Hirsch JM, Rannar LE, Derand P, Omar O (2016) Clinical, morphological, and molecular evaluations of bone regeneration with an additive manufactured osteosynthesis plate. J Craniofacial Surg 27:1899-1904

7. Bonewald LF (2011) The amazing osteocyte. J Bone Miner Res $26: 229-238$

8. Janner SF, Bosshardt DD, Cochran DL, Chappuis V, Huynh-Ba G, Jones AA, Buser D (2016) The influence of collagen membrane and autogenous bone chips on bone augmentation in the anterior maxilla: a preclinical study. Clin Oral Implants Res. doi:10.1111/clr.12996

9. Franchi M, Bacchelli B, Martini D, Pasquale VD, Orsini E, Ottani V, Fini M, Giavaresi G, Giardino R, Ruggeri A (2004) Early detachment of titanium particles from various different surfaces of endosseous dental implants. Biomaterials 25:2239-2246

10. Franchi M, Fini M, Martini D, Orsini E, Leonardi L, Ruggeri A, Giavaresi G, Ottani V (2005) Biological fixation of endosseous implants. Micron 36:665-671

11. Shah FA, Wang X, Thomsen P, Grandfield K, Palmquist A (2015) High-resolution visualization of the osteocyte lacunocanalicular network juxtaposed to the surface of nanotextured titanium implants in human. ACS Biomater Sci Eng 1:305-313

12. Shipov A, Zaslansky P, Riesemeier H, Segev G, Atkins A, Shahar R (2013) Unremodeled endochondral bone is a major architectural component of the cortical bone of the rat (Rattus norvegicus). J Struct Biol 183:132-140

13. Shah FA, Zanghellini E, Matic A, Thomsen P, Palmquist A (2016) The orientation of nanoscale apatite platelets in relation to osteoblastic-osteocyte lacunae on trabecular bone surface. Calcif Tissue Int 98:193-205

14. Barragan-Adjemian C, Nicolella D, Dusevich V, Dallas MR, Eick JD, Bonewald LF (2006) Mechanism by which MLO-A5 late osteoblasts/early osteocytes mineralize in culture: similarities with mineralization of lamellar bone. Calcif Tissue Int 79:340-353

15. Hernandez CJ, Majeska RJ, Schaffler MB (2004) Osteocyte density in woven bone. Bone 35:1095-1099

16. Elgali I, Igawa K, Palmquist A, Lennerås M, Xia W, Choi S, Chung UI, Omar O, Thomsen P (2014) Molecular and structural patterns of bone regeneration in surgically created defects containing bone substitutes. Biomaterials 35:3229-3242

17. Roberts TT, Rosenbaum AJ (2012) Bone grafts, bone substitutes and orthobiologics: the bridge between basic science and clinical advancements in fracture healing. Organogenesis 8:114-124

18. Hutchison J (1952) The fate of experimental bone autografts and homografts. Br J Surg 39:552-561

19. Shah FA, Johansson ML, Omar O, Simonsson H, Palmquist A, Thomsen P (2016) Laser-modified surface enhances osseointegration and biomechanical anchorage of commercially pure 
titanium implants for bone-anchored hearing systems. PLoS ONE 11:e0157504

20. Shah FA, Nilson B, Brånemark R, Thomsen P, Palmquist A (2014) The bone-implant interface-nanoscale analysis of clinically retrieved dental implants. Nanomedicine 10:1729-1737
21. Franssen BB, van Diest PJ, Schuurman AH, Kon M (2008) Drilling K-wires, what about the osteocytes? An experimental study in rabbits. Arch Orthop Trauma Surg 128:83-87

22. Eriksson AR, Albrektsson T (1983) Temperature threshold levels for heat-induced bone tissue injury: a vital-microscopic study in the rabbit. J Prosthet Dent 50:101-107 